\section{Research News}

\section{Door to Ebola}

The folate receptor has been shown to have a far more diabolical function than just transporting folic acid into cells. In the 13 July issue of Cell, Chan et al. report that Ebola and Marburg filoviruses, which cause hemorrhagic fever, apparently use it to enter an extensive range of mammalian cell types. Filovirus entry is mediated by binding of a transmembrane envelope glycoprotein to an unknown cell-surface receptor. The authors used a complementation screen of $\mathrm{T}$ cells, which are naturally resistant to infection, to search for a gene encoding a filovirus receptor. A cDNA encoding the folate receptor- $\alpha$ (FR- $\alpha$ ) was able to reconsitute entry of both Marburg and Ebola virus into T cells, whereas FR- $\alpha$ blocking agents inhibited infection. FR- $\alpha$ is highly conserved in a number of mammalian species, corresponding with the wide tropism of filoviruses. The receptor is highly expressed in a variety of epithelial and parenchymal cells, as well as macrophages and fibroblasts, the major targets of filoviruses. Marburg and Ebola viruses share a common pathway for infection, and agents designed to block FR- $\alpha$ may be developed as antivirals.

\section{Overcoming the pain of rejection}

Chronic renal-transplant rejection involves tissue remodeling events such as fibrointimal hyperplasia and interstitial fibrosis. These processes are mediated by mesenchymal cells such as smooth-muscle cells and myofibroblasts. It was long believed that the mesenchymal cells that cause this damage arise from the allograft itself. In the 12 July issue of NEJM, Grimm et al. investigated the origin of mesenchymal cells using sex-mismatched renal allografts from patients undergoing chronic rejection. In male hosts receiving renal allografts from a female donor, a vast majority of of mesenchymal cells (identified by $\alpha$-actin staining) in the neointima, adventitia and tubointerstitial compartments contained the Y-chromosome, indicating that they originated from the recipient. Examination of additional renal allografts in which the donor was male and the recipient was female also revealed a persistent population of donor mesenchymal cells. Grimm et al. suggest that circulating mesenchymal precursor cells have the potential to colonize an allograft. Smooth-muscle-cell proliferation and migration are regulated by hyaluronan signaling through CD44, and agents designed to interfere with this pathway might prevent chronic rejection.

\title{
Hedgehog troubles
}

Geneticists have identified defects in a gene encoding a hedgehog signaling protein as the cause of Brachydactyly type A-1 (BDA-1), a disease characterized by short stature and shortening or missing of the middle phalanges (Picture). BDA-1, the first recorded example of a human disorder with mendelian autosomal-dominant inheritance, was recently mapped to a region of
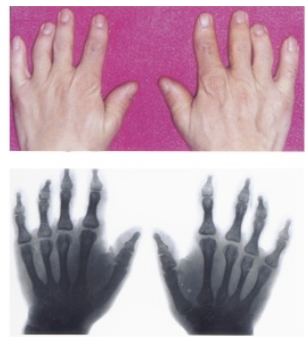

domain. The hedgehog family of proteins, which includes the genes encoding desert hedgehog and sonic hedgehog, are involved in vertebrate chromosome 2q35-q36. In the August issue of Nature Genetics, Gao et al. report that mutations in the gene encoding Indian hedgehog $(I H H)$, which is located in this region, underlie BDA-1. Affected members of three large unrelated families were found to carry missense mutations in the $I H H$ region encoding the amino-terminal signaling and invertebrate limb development. Gao et al. suggest that the mutations interfere with the protein's signaling ability by disrupting receptor binding. Indian hedgehog is known to mediate condensation, growth and differentiation of cartilage, and mice carrying disruptions in the Ihh gene have shortened forelimbs and unsegmented, uncalcified digits. Further study of these three families might provide further insight into mechanisms of Indian hedgehog signaling and limb development.

\section{Pneumonia researchers can breathe a sigh of relief...}

now that the genome of Streptococcus pneumoniae has been sequenced. S. pneumoniae, the bacteria that causes pneumonia, bacteremia and meningitis, leads to the death of 3 million children and even more elderly people each year. In the 20 July issue of Science, Tettelin et al. report the complete sequence of a clinical isolate of the pathogen. The genome contains 2,236 coding regions, $64 \%$ of which can be assigned a biological function. S. pneumoniae possess many genes involved in sugar metabolism and more genes encoding sugar transporters than other respiratory tract pathogens. The authors conclude that $S$. pneumoniae might therefore occupy a distinct environment within the respiratory tract. The study also identified many sequence differences among strains of the bacterium that could underlie differences in virulence. Several surface-exposed proteins were also identified, which can be developed as vaccine targets. Further sequence analysis will help scientists understand the factors that make the bacterium so virulent, and also help them to understand why certain strains have recently become resistant to antibiotics.

\section{Liver savers}

Prostaglandins have well-known roles in the inflammatory response, but a recent study demonstrates that they are also required for liver regeneration. Prostaglandins are signaling molecules involved in regulating cell proliferation in a wide variety of tissues, and their synthesis requires the enzymes cyclo-oxegenase 1 (COX1) and COX2. In the 17 July issue of PNAS, Rudnick et al. use a mouse partial hepatectomy model to show that liver regeneration is impaired when COX-1 or COX-2 activity is inhibited either pharmacologically or by gene disruption. Prostaglandins are involved in the regulation of several cytokines associated with liver regeneration, including tumor necrosis factor- $\alpha$ and interleukin6 . However, neither production of these cytokines nor activation of their downstream factor STAT3 was affected by COX enzyme inhibition. These results indicate that prostaglandin signaling occurs through cytokine-independent pathways during liver regeneration. In their study, Rudnick et al. showed that after hepatectomy, COX inhibitors interfere with the activation of cAMP-mediated signaling pathways involving the transcription factor CREB. Using specific inhibitors of COX-1 and COX-2, the authors also demonstrate that these enzymes each have distinct roles in the hepatic cell response to liver damage. COX-1 and COX-2 were recently shown to have similarly distinct functions in induction of angiogenesis.

\section{By Kristine Novak}

